\title{
A new device laboratory: Experimental validation *
}

\author{
G. Pujol ${ }^{*}$ L. Acho ${ }^{*}$ A. Nápoles ${ }^{* *}$ J. Estrada ${ }^{* *}$ D. Santos ${ }^{* *}$ \\ V. Pérez-García ${ }^{* * *}$ \\ * Department of Applied Mathematics III, Universitat Politècnica de \\ Catalunya-BarcelonaTech (EUETIB), Comte Urgell 187, 08036 \\ Barcelona, Spain. (email: gisela.pujol@upc.edu, \\ leonardo.acho@upc.edu). \\ ** Department of Mechanical Engineering, Universitat Politècnica de \\ Catalunya-BarcelonaTech (EUETIB), Comte Urgell 187, 08036 \\ Barcelona, Spain.(email: amelia.napoles@upc.edu). \\ *** Department of Strength of Materials and Structural Engineering, \\ Universitat Politècnica de Catalunya-BarcelonaTech (EUETIB), \\ Comte Urgell 187, 08036 Barcelona, Spain. (email: \\ vega.perez@upc.edu).
}

\begin{abstract}
A device laboratory was designed to create a commanded disturbance to the Furuta inverted pendulum. This pendulum was modified by adding a second inverted pendulum coupled to the main one by means of a semi-rigid spring. The induced motion on the second inverted pendulum causes displacement of the center of mass of the system, producing a kind of perturbation similar to that presented on mobile inverted pendulum transportation units. A linear matrix inequality (LMI) controller is designed from the unperturbed model (based on the main pendulum without the second inverted one) and implemented to our system. Then, experimentally, the behaviour of the whole closed-loop system and the controller performance was analysed. According to the laboratory test, the LMI controller is robust enough in front of perturbation induced on the second pendulum.
\end{abstract}

Keywords: Robust control, LMI, Device Laboratory.

\section{INTRODUCTION}

We develop a control engineering experiment for use by graduate students in learning about process control. The commanded inverted pendulum system has been built as an educational kit for control education in mechanical grade, where students leave the university with little exposure to common control theory.

Under-actuated dynamical systems are those that have more degrees-of-freedom than control inputs. Examples include spacecrafts, underwater autonomous vehicles and mobile robots. Control and stabilisation of these systems are challenging tasks and are currently hot topics of research for both engineers and applied mathematicians. New stabilisation strategies are validated and tested on classical benchmark systems such as the 'ball on a beam' and 'inverted pendulum' systems (Astrom et al. 2000, Awtar et al. 2002). An interesting problem comes from in troducing some kind of perturbation to these dynamical systems, to study the robustness in front external disturbances (Pujol et al. 2010). Also, one such systems which has drawn the attention of control researchers is the Mobile

\footnotetext{
* This work was partially funded by the Spanish Government, the European Commission, and FEDER funds, through the research projects: CGL2008-00869/BTE, CGL2011-23621, DPI2011-26326, DPI2011-25822 and DPI2012-32375.
}

Inverted Pendulum (MIP) which is a two-wheeled robot with a central body that carries a payload. The robot has the advantage of having a small footprint in addition to its ability to turn about its central axis. A commercial variant of the MIP is the well known Segway (Nguyen et al. 2004). We can define a Segway Robotic Mobile Platform (RMP) as self-balancing personal transport unit which is similar to the classic control inverted pendulum stabilisation problem. The navigation of this unit is due to, basically, the induced displacement of the center mass of the system.

Following this line of reasoning, we mechanically modified the Furuta pendulum (Fig. 1), by adding a second inverted pendulum coupled to the main one (see Fig. 2 and 3). With it, the center mass position of the second inverted pendulum is modified by radio control, to induce a perturbation which produces displacement of the Furuta pendulum. In practice, we have modified an existent experiment Furuta pendulum presented in Figures 1 and 5 (provided by ECP-systems) by replacing weight $m_{w}$ by this second inverted pendulum. This new experiment is coupled inverted pendulums (due to the two pendulums). Figures 2 and 3 present this new experiment realisation. This external perturbation (second pendulum) is quantised by measuring its angular position via an encoder 
from Passport company. So, we can study directly how this disturbance actuates on our inverted pendulum.

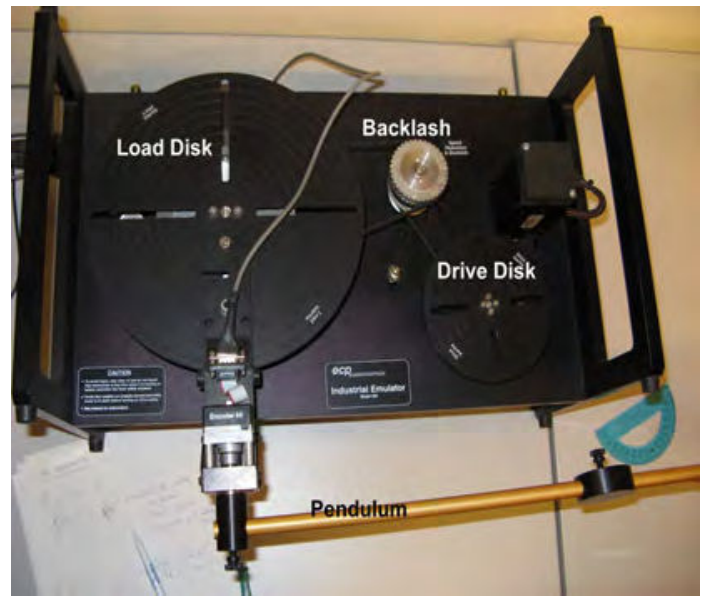

Fig. 1. Basic pendulum assembly control (Model M220) of ECP systems company (ECP 2003).

Our control objective is to experiment with this dynamic system in order to prove robust stability on a Linear Matrix Inequality (LMI) controller. Many nonlinear results exist in the bibliography, incorporating experimental applications (Sekhavat et al. 2004, Tao et al. 1996, Tao et al. 2003), but the purpose of the present paper is to present an easy control algorithm that solves a complex nonlinear problem. For linear systems, $H_{\infty}$ control theory offers the possibility of including robustness considerations explicitly in the design and the opportunity to formulate physically meaningful performance objectives that can be expressed as $H_{\infty}$ design specifications (Doyle et al. 1989, Khargonekar et al. 1990), and solved via the LMI techniques (Oliveira et al. 2006, Apkarian et al. 2001). It is convenient to point that firstly the $H_{\infty}$ linear controller clammed in (Doyle et al. 1989) (in the output feedback case) was implemented in our Furuta experiment, but experimentally the closed-loop systems was unstable (Pujol et al. 2010). In this paper, a LMI controller was obtained from the un-perturbed model (the one without the second inverted pendulum), and the controller performance was evaluated experimentally. According to experiments, the LMI controller is robust against this kind of perturbation. In particular, its possible realisation as a Segway navigation control unit center was proved.

This paper presents a coupled pendulum control system. Section two discuss the Segway realisation. Then, mathematical model as well as the control problem are described in Sections three. Experimental test and results are commented in Section four, showing controller robustness. Finally, Section five gives the conclusions.

\section{DEVICE LABORATORY DESIGN: SEGWAY INSPIRATION}

As it was said in the introduction, under-actuated dynamical systems are those that have more degrees-of-freedom than control inputs. Examples include spacecrafts, underwater autonomous vehicles and mobile robots. One such systems which has drawn the attention of control researchers is the mobile inverted pendulum (MIP) which
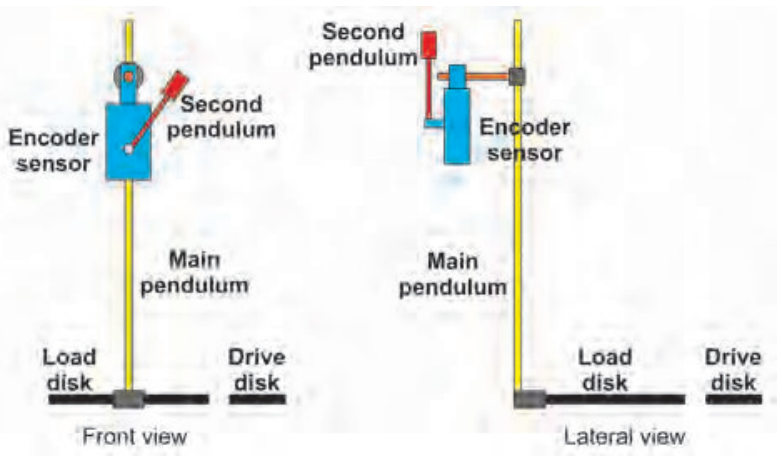

Fig. 2. Coupled pendulums experiment: A new realisation (front and lateral view).

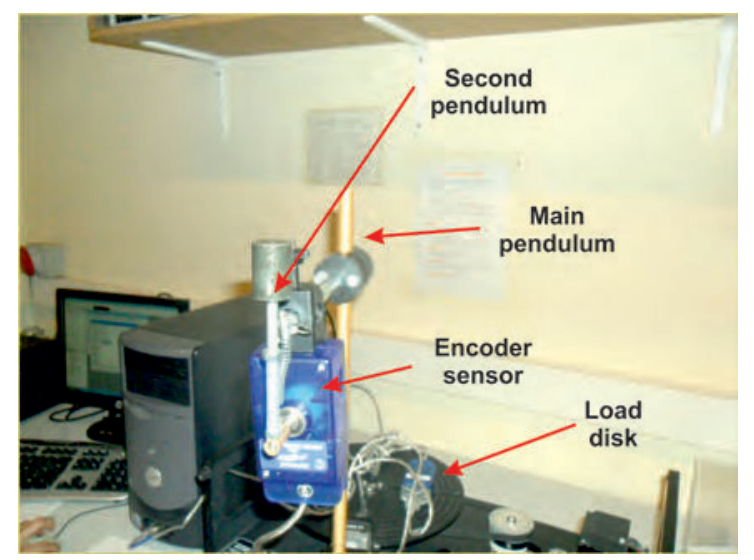

Fig. 3. Coupled pendulums experiment: A new realisation. On this picture, in blue, the encoder sensor. In gold, the main pendulum. In red, the second pendulum coupled to the main one.

is a two-wheeled robot with a central body that carries a payload. The robot has the advantage of having a small footprint in addition to its ability to turn about its central axis. A commercial variant of the MIP is the well known Segway (Nguyen et al. 2004). Based on LMI techniques, (Azizan et al. 2010) presents a hierarchy intelligent control scheme for a Segway vehicle, but without real experimentation. Also, a fuzzy control is designed and implemented to the same problem in (Huang et al. 2011). We can define a Segway Robotic Mobile Platform (RMP) as selfbalancing personal transport unit which is similar to the classic control inverted pendulum stabilisation problem. The navigation of this unit is due to, basically, the displacement of the center mass of the body. This line of reasoning motivates us our design showed in Figures 32. With this new device, the center mass position of the second inverted pendulum is modified by radio control, to induce a perturbation which produces displacement of the Furuta pendulum. At this respect, the principal pendulum, attached to the rotating base (Fig. 1, 2, 3 and 4), can emulate the Segway machine, with the difference that the Segway moves linearly, but the Furuta pendulum rotates. The second inverted pendulum can emulate a person (Fig. 4). 

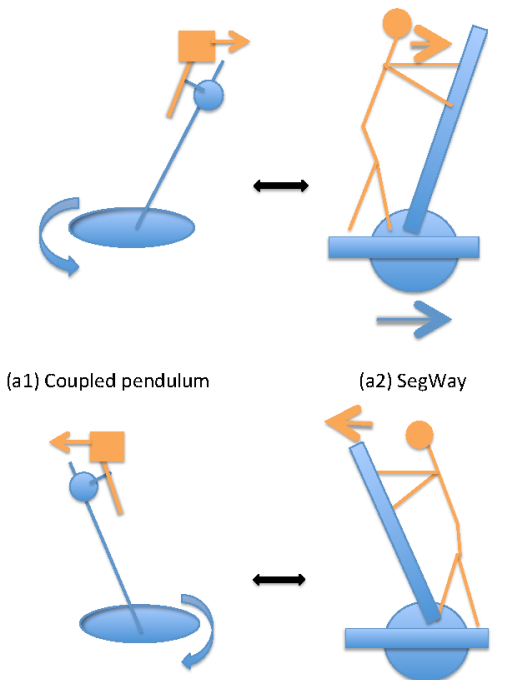

(a2) SegWay

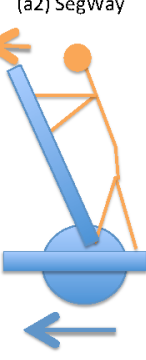

(b1) Coupled pendulum

(b2) SegWay

Fig. 4. Basic Segway inspiration scheme.

\section{DYNAMICAL SYSTEM EQUATIONS AND CONTROL STATEMENT}

\subsection{Dynamical system equations}

The rotating base system driven by a motor shown in Figures 1 and 5 is representative of the pendulum attached to experimental Model 220 apparatus (ECP 2003), where $\theta_{1}$ is the magnitude of angular rotation of the disk with respect to $O_{1}$ and $\theta_{2}$ is the magnitude of angular rotation of the pendulum with respect to $\mathrm{O}_{2}$. In the case study described in this paper, the mass set at $P$ (weight $m_{w}$ in Fig. 5) is changed by a second inverted pendulum with the same mass (Fig. 3-2). Both pendulums are coupled with a semi-rigid spring.
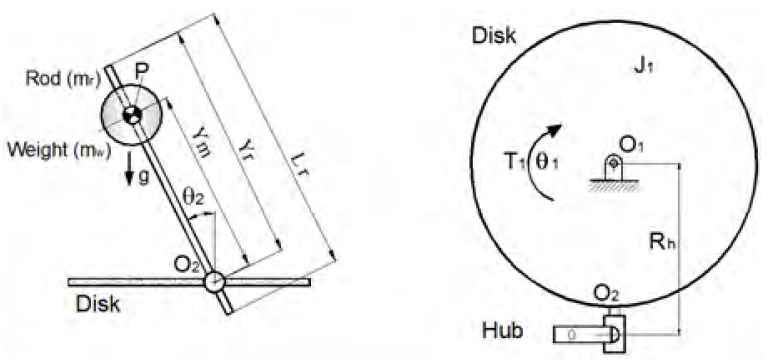

Fig. 5. Diagram representation of Furuta pendulum with rotating base (ECP 2003); $J_{1}$ includes equivalent inertia of all elements that move uniformly with the disk.

The equations of motion for the un-perturbed case are obtained from Lagrange's equations and may be linearised in the case of $\theta_{1}=0$ and $\theta_{2}=0(\operatorname{ECP} 2003)$ :

$$
\ddot{\theta}_{1}=\frac{1}{p}\left(-\dot{\theta}_{1} \bar{J}_{z}-m^{2} l_{c g}^{2} R_{h} g \theta_{2}+\bar{J}_{z} u\right)
$$

$$
\ddot{\theta}_{2}=\frac{1}{p}\left(m R_{h} l_{c g} \dot{\theta}_{1}-m g l_{c g}\left(\bar{J}_{1}+J_{y}\right) \theta_{2}-m R_{h} l_{c g} u\right),
$$

where $p=\bar{J}_{z}\left(\bar{J}_{1}+J_{y}\right)-\left(m R_{h} l_{c q}^{2}\right), \bar{J}_{1}=J_{1}+m R_{h}^{2}$ and $\bar{J}_{z}=J_{z}+m l_{c g} ; J_{1}$ includes equivalent inertia of all elements that move uniformly with the motor disk; $J_{y}$ and $J_{z}$ are the pendulum moments of inertia, relative to its center of mass; $l_{c g}$ is the center of gravity of the combined pendulum road and weight $\left(m=m_{r}+m_{w}\right) ; g$ is the gravity constant; and $u(t)$ is the control effort. For more details on parameters and modelling, see (ECP 2003). In this paper, the state vector is $\mathbf{x}(t)=\left[\begin{array}{llll}\theta_{1} & \dot{\theta}_{1} & \theta_{2} & \dot{\theta}_{2}\end{array}\right]$ and $u(t)=\mathbf{K x}(t)$. A linear state-feedback strategy is adopted to simplify the approach. In fact, it is convenient to point that firstly the $H_{\infty}$ linear controller clammed in (Doyle et al. 1989) (in the output feedback case) was implemented in our Furuta experiment. Notwithstanding, the experimental test indicates that the closed-loop system was unstable.

Only the load disk angle position $\left(\theta_{1}\right)$ and the pendulum angle position $\left(\theta_{2}\right)$ are available by the experimental platform, but velocities will be required in the control design; so, an observer is constructed to estimate the velocities $\dot{\theta_{1}}$ and $\dot{\theta_{2}}$. The angular position of the second pendulum is measured off-line from the control-loop via an encoder.

\subsection{Control objective}

The control aim of this work is to design a robust control verifying two properties. One is to ensure local stability. The other is the requirement imposed to the control design to be robust in front of $\mathcal{L}_{2}$ disturbance. The problem of robust controller with guaranteed $H_{\infty}$ performance is addressed to answer this question: Does there exist a feedback control such that the $H_{\infty}$ norm of the closed-loop system from input disturbance named $w(t)$ to output $z(t)$ is less than some prescribed value $\gamma$ ? (Doyle et al. 1989). In order to solve this problem, the linear matrix inequalities (LMI) techniques are used.

Let us consider system (1)-(2) where the control effort is the effective control $u(t)$. By model variables stated previously, the vector state is defined as $\mathbf{x}^{T}(t)=$ $\left[\begin{array}{llll}\theta_{1}(t) & \dot{\theta}_{1}(t) & \theta_{2}(t) & \dot{\theta}_{2}(t)\end{array}\right]$. Consider $\mathbf{z}^{T}(t)=\left[\begin{array}{lll}\theta_{1} & \theta_{2} & u\end{array}\right]$ the virtual output to be compared to the $\mathcal{L}_{2}$ perturbation $w(t)$ (induced by the second pendulum). Then, the statespace representation of system (1)-(2) yield

$$
\left\{\begin{array}{l}
\dot{\mathbf{x}}(t)=\mathbf{A} \mathbf{x}(t)+\mathbf{B}_{2} u(t)+\mathbf{B}_{1} w(t) \\
\mathbf{z}(t)=\mathbf{C}_{\mathbf{1}} \mathbf{x}(t)+\mathbf{D}_{12} u(t)
\end{array}\right.
$$

where

$$
\mathbf{A}=\frac{1}{p}\left[\begin{array}{cccc}
0 & 1 & 0 & 0 \\
0 & -\bar{J}_{z} & -m^{2} l_{c g}^{2} R_{h} g & 0 \\
0 & 0 & 0 & 1 \\
0 & m R_{h} l_{c g} & m l_{c g} g\left(\bar{J}_{1}+J_{y}\right) & 0
\end{array}\right],
$$




$$
\begin{gathered}
\mathbf{B}_{1}=\mathbf{B}_{2}=\frac{1}{p}\left[\begin{array}{c}
0 \\
\bar{J}_{z} \\
0 \\
-m R_{h} l_{c g}
\end{array}\right], \\
\mathbf{C}_{1}=\left[\begin{array}{llll}
1 & 0 & 0 & 0 \\
0 & 0 & 1 & 0 \\
0 & 0 & 0 & 0
\end{array}\right] \text { and } \mathbf{D}_{12}=\left[\begin{array}{l}
0 \\
0 \\
1
\end{array}\right] .
\end{gathered}
$$

Furthermore, the above dynamic model satisfies the following standard ${ }^{1} H_{\infty}$ assumptions (Doyle et al. 1989):

(1) $\left(\mathbf{A}, \mathbf{B}_{1}\right)$ is stabilisable and $\left(\mathbf{C}_{1}, \mathbf{A}\right)$ detectable;

(2) $\left(\mathbf{A}, \mathbf{B}_{2}\right)$ is stabilisable;

(3) $\mathbf{D}_{12}^{T}\left[\begin{array}{ll}\mathbf{C}_{1} & \mathbf{D}_{12}\end{array}\right]=\left[\begin{array}{ll}0 & I\end{array}\right]$.

Our control objective is to design a gain matrix $\mathbf{K}$ such that the controller

$$
u(t)=\mathbf{K x}(t),
$$

stabilises the system $(3)$ under $\mathcal{L}_{2}$ disturbances, employing $H_{\infty}$-LMI theory. This case has been named fullinformation problem ((Doyle et al. 1989)). A practical way to solve this problem is to consider a Lyapunov function $V(\mathbf{x}(t))$ such that for any nonzero $\mathbf{x}(t)$ and input $w(t) \in$ $\mathcal{L}_{2}$, the following condition holds ((Apkarian et al. 2001)):

$$
\frac{d}{d t} V(\mathbf{x}(t))+\gamma^{-1} \mathbf{z}^{T}(t) \mathbf{z}(t)-\gamma w^{T}(t) w(t)<0 .
$$

Then, an $H_{\infty}$ performance bound for the closed-loop system (3)-(4) is ensured (see (Apkarian et al. 2001) for details). If there exists a matrix $\mathbf{K}$ such that (5) holds, then the control law $u(t)=\mathbf{K} \mathbf{x}(t)$ is said to be an $H_{\infty}$ controller for the system (3), that is, the system is internally stable with $H_{\infty}$ norm less than $\gamma$, i.e., $\|\mathbf{z}\|_{\infty} \leq$ $\gamma^{2}\|w\|_{\infty}$ for $w \in \mathcal{L}_{2}$.

The control design for solving the $H_{\infty}$ problem (5) involves two steps. First, a sufficient condition is obtained for the existence of an $H_{\infty}$ controller, in terms of a nonlinear matrix inequality. The second step is based on deriving an LMI procedure for the controller effective construction. As instrumental tool, a result based on Projection Lemma $(($ Gahinet et al. 1994)) is needed, where an LMI characterisation is stated.

Theorem 1. ( $H_{\infty}$ Control). Consider the Furuta pendulum system (3)-(4). If there exist $\gamma>0$, matrices $\mathbf{N}, \mathbf{Y}>0$ symmetric and $\mathbf{V}$ regular such that the LMI

$$
\left[\begin{array}{cccccc}
-\left(\mathbf{V}^{T}+\mathbf{V}\right) & * & * & * & * & * \\
\mathbf{A V}+\mathbf{Y}+\mathbf{B}_{2} \mathbf{N} & -\mathbf{Y} & * & * & * & * \\
0 & \mathbf{B}_{1}^{T} & -\gamma & * & * & * \\
\mathbf{C}_{1} \mathbf{V} & 0 & 0 & -\gamma & * & * \\
\mathbf{N} & 0 & 0 & 0 & -\gamma & * \\
\mathbf{V} & 0 & 0 & 0 & 0 & -\mathbf{Y}
\end{array}\right]<0
$$

\footnotetext{
1 Instead of the four standard assumptions stated in (Doyle et al. 1989), one of them is relaxed because it is not required in our control design scheme.
}

is feasible, then the inequality (5) holds, with Lyapunov function defined as $V(x)=x^{T} \mathbf{P} x$ with $\mathbf{P}:=\mathbf{Y}^{-1}$, and the control $u(t)=\mathbf{K x}(t)$ with $K:=N V^{-1}$. Consequently, $u(t)$ is an $H_{\infty}$ controller.

The proof of this result is straightforward from (Pujol et al. 2010).

\section{EXPERIMENTS TO AN INDUSTRIAL EMULATOR}

To test the obtained theoretical results, the controller effectiveness is studied experimentally. We design the control (4) via the resolution of the LMI state in Theorem 1. Experiments have been performed on an ECP Model 220 industrial emulator with Furuta pendulum that includes a PC-based platform and DC brushless servo system (ECP 2003).

The mechatronic system includes a motor used as servo actuator, a power amplifier and two encoders which provide accurate position measurements; i.e., 4000 lines per revolution with $4 X$ hardware interpolation giving 16000 counts per revolution to each encoder; 1 count (equivalent to 0.000392 radians or 0.0225 degrees) is the lowest angular measurable (ECP 2003). The second pendulum (external disturbance) includes an angular position sensor measured in radians. The drive and load disks were connected via a $4: 1$ speed reduction (Fig. 1).

In the experiments, the pendulum is set to the following parameters: $y_{r}=42 \mathrm{~cm}, y_{m}=32 \mathrm{~cm}$ (Fig. 5).The parameters were taken from (ECP 2003):

$$
\begin{gathered}
\mathbf{A}=\left[\begin{array}{cccc}
0 & 1 & 0 & 0 \\
0 & -1.1379 & -28.769 & 0 \\
0 & 0 & 0 & 1 \\
0 & 0.7219 & 50.229 & 0
\end{array}\right], \\
\mathbf{B}_{1}=\mathbf{B}_{2}=\left[\begin{array}{c}
0 \\
318.7 \\
0 \\
-202.2
\end{array}\right]
\end{gathered}
$$

Note that the perturbation and the control law enter to the system by the same channel $\left(B_{1}=B_{2}\right)$. Using the technique presented in Theorem 1 and solving (6) with the LMI Matlab Toolbox (Chiang et al. 1998), the next $H_{\infty}$ sub-optimal controller was obtained:

$$
\mathbf{K}=\left[\begin{array}{llll}
0.38 & 0.43 & 6.38 & 1.09
\end{array}\right],
$$

with $\gamma=8.2$. This expression may be used directly for control modelling, scaled by appropriate system gains (amplifier and software gains and motor torque constants). In the experiments, the controller in (7) was multiplied by 0.3 to compensate these gains.

Because velocity measurements are not available, the following controller realisation is developed, where the velocity part is replaced by a first-order linear compensator:

$$
\begin{aligned}
& u=0.3\left(0.38 \theta_{1}+0.43 \dot{x}_{1}+6.38 \theta_{2}+1.09 \dot{x}_{2}\right), \\
& \dot{x}_{1}=-10 x_{1}+5 \theta_{1}, \\
& \dot{x}_{2}=-10 x_{2}+5 \theta_{2} .
\end{aligned}
$$

The terms $x_{1}$ and $x_{2}$ are auxiliary variables and their differential equations are solved numerically from $\theta_{1}$ and 
$\theta_{2}$ measurements. The above equations are a direct implementation of velocity observers given in (Berghuis et al. 1993), where the parameter -10 was set according to (Ogata 1997) and the gain 5 was adjusted experimentally ${ }^{2}$. This approach goes along a two independent steps design procedure: a) design a state-feedback controller $K$ as a complete access to state vector is available, and b) construct a velocity observer. This design obeys the separation principle (see (Ogata 1997) and (Khalil 2000)).

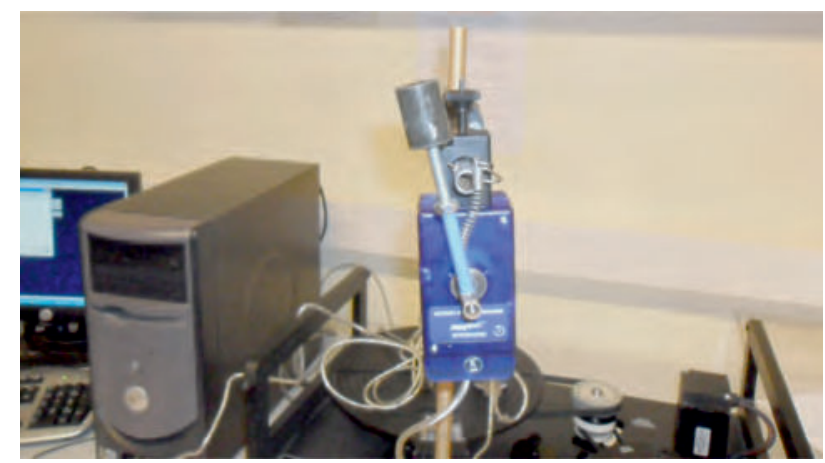

Fig. 6. External disturbance: First position modification of pendulum accessory, at 17 sec. (see Fig. 8)

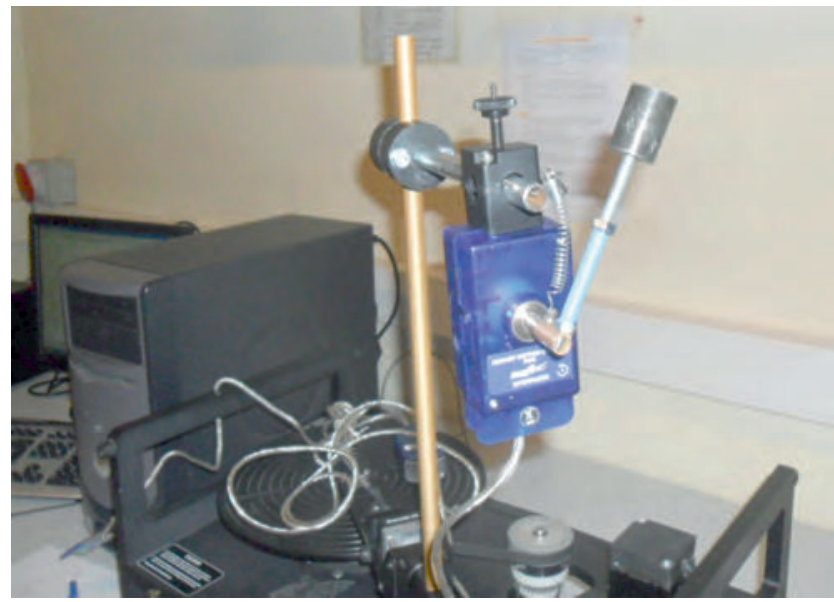

Fig. 7. External disturbance: Second position modification of pendulum accessory, at 35sec. (see Fig. 8)

Figures 6 and 7 present two different positions on the pendulum. These positions were realised by radio control during the experiment ( 57 sec.). Figure 8 pictures the exact perturbation produced by this second inverted pendulum, measured with the angular position encoder referred to the vertical. As Fig. 8 shows, the initial position is not exact the zero position. Also, in Fig. 9, we can appreciate that the initial position of the inverted pendulum is not exactly zero, inducing a small vibration (50 Counts $=0.0196$ radians). This is due to the difficulty on starting the system exactly at the equilibrium point.

Figures 9-11 present the time history plots of the drive disk, load disk (where pendulums are located), principal pendulum positions, and the control effort, respectively.

2 This consist to locate the observer poles from 5 to 10 times far away with respect to the vertical-axis-closest pole of $\left(A+B_{2} K\right)$.

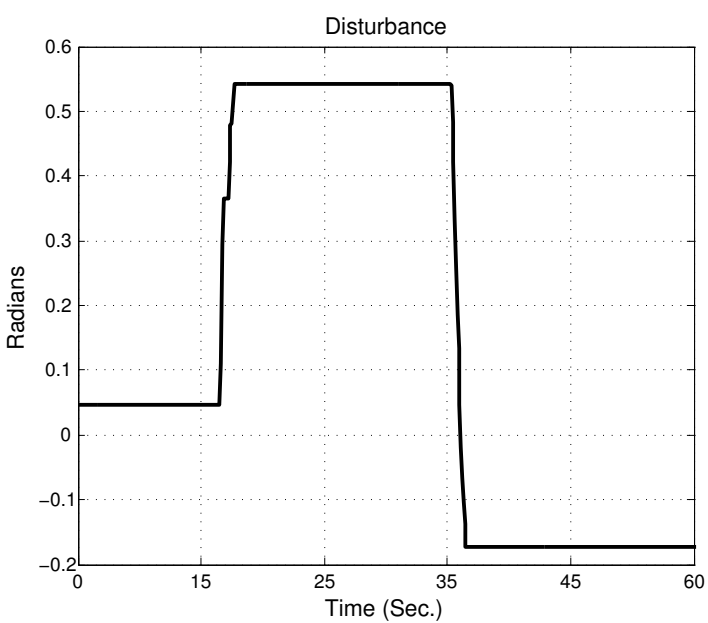

Fig. 8. External disturbance produced by the pendulum accessory, measured via the Passport encoder.

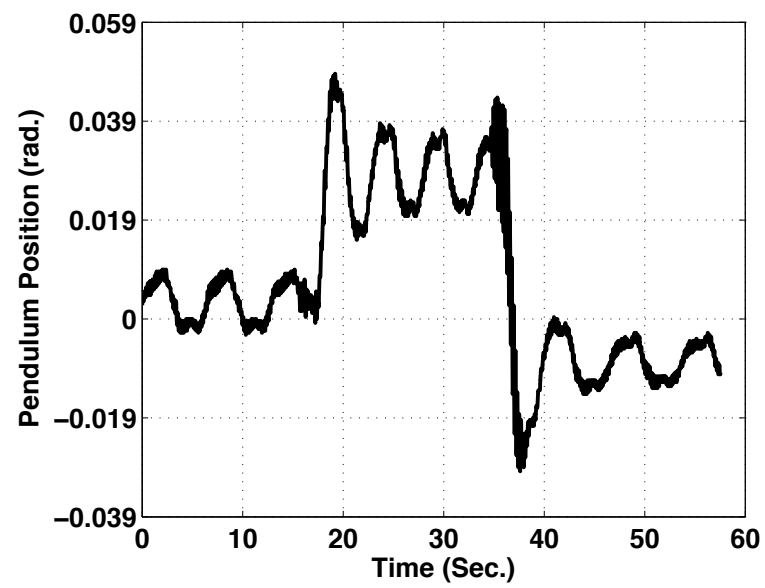

Fig. 9. Pendulum angular position.

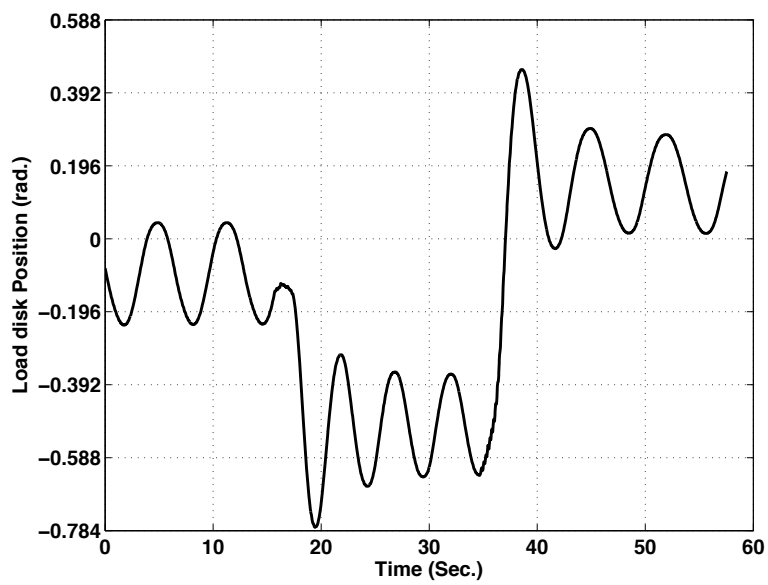

Fig. 10. Load disk angular position, where the pendulums are located (see Fig. 1).

These figures show the robustness of the proposed controller (7), and the efficiency of the method presented in Theorem 1. In the graphs Fig. 8-11, it could be observed that the changes in the control effort corresponds to the start and end of the perturbation signal, being maximum 


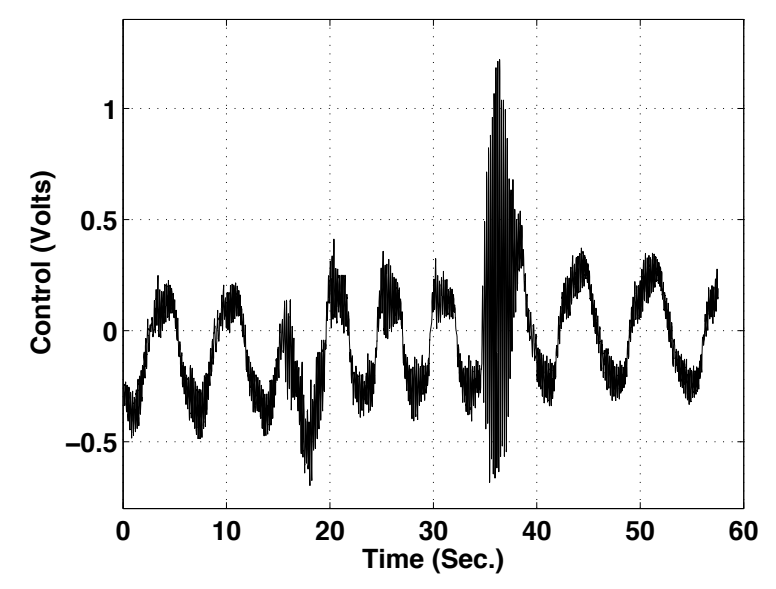

Fig. 11. Control effort.

in the case of greater disturbance. However, a 'quick' return to the stable equilibrium region is observed.

\section{CONCLUSION}

A new experimental set up for the Furuta pendulum was developed to validate controller performance under a kind of mass perturbation similar to the one used to navigate the Segway personal transportation unit. To simulate the Segway behaviour, a second pendulum was elastically coupled to the main one. The control was designed in order to ensure stability and robustness. Experimental results demonstrate that the objectives have been achieved. Hence, the mechanical experiment provides interesting new results in the control automatic new developments.

\section{REFERENCES}

K.L. Åström and K. Furuta. Swinging up a pendulum by energy control. Automatica, volume 36:287-295, 2000.

S. Awtar, N. King, T. Allen, I. Bang, M. Hagan, D. Skidmore, and K. Graig. Inverted pendulum systems: rotary and arm-driven, a mechatronic system design case study. Mechatronics, volume 12:357370, 2002.

G. Pujol and L. Acho. Stabilization of the Furuta pendulum with backlash using $H_{\infty}$-LMI technique: Experimental validation. Asian Journal of Control, volume 12(4):460-467, 2010.

H.G. Nguyen, J. Morrell, K. Mullens, A. Burmeister, S. Miles, N. Farrington, K. Thomas, and D.W. Gagee. Segway robotic mobility platform. SPIE Proceedings 5609: Mobile Robots XVII, Philadelphia, PA, October 27-28, 2004.

Sekhavat P., Wu Q., Sepheri N. Lyapunov-based Friction Compensation for Accurate Positioning of a Hydraulic Actuator, Proc. of the 2004 American Control Conference, boston, U.S.A., 2004.

Tao G., Kokotovic P. Adaptive control system with actuator and sensor nonlinearities, Wiley InterScience, 1996.

Taware A., Tao G., Control of sandwich nonlinear systems, Lecture notes in control and information sciences, Springer, 2003.
J.C. Doyle, K. Glove, P. Khargonekar, and B.A. Francis. State-space solutions to standard $H_{2}$ and $H_{\infty}$ control problems. IEEE Transaction on Automatic Control, volume 34(8):831-847, 1989.

Khargonekar P., Petersen I., Zhou K., Robust stabilization of uncertain linear systems: quadratic stabilization and $H_{\infty}$ control theory, IEEE Tran. on Aut. Control, Vol. 35, No. 3, pp.356-361, 1990.

Oliveira R.C.L.F., Peres P.L.D., "LMI conditions for robust stability analysis based on polynomial parameter-dependent Lyapunov functions", Systems and Control Letters, Vol. 55, No.1, pp.5261, 2006.

P. Apkarian, H.D. Tuan, and J. Bernussou. Continuostime analysis, eigenstructure assignment and $H_{2}$ synthesis with enhanced LMI characterizations. IEEE Transaction on Automatic Control, volume 46(12):1941-1946, 2001.

ECP Model. Manual for A-51 inverted pendulum accessory (Model 220). Educational Control Products, California 91307, USA, 2003.

P. Gahinet and P. Apkarian. A linear matrix inequality approach to $H_{\infty}$ control. International Journal of Nonlinear Control, volume 4:421-448, 1994.

R. Y. Chiang, and M. G. Safonov. Matlab Robust Control Toolbox User's Guide version 2. The MathWorks Inc., MA, USA, 1998.

Berghuis H., Nijmeijer H. Global regulation of robots using only position measurements. Systems and Control Letters, volume 21:289-293,1993.

K. Ogata. Modern control engineering. Prentice-Hall ed., chapter 12, New York, 1997.

H.K. Khalil. Nonlinear systems Prentice-Hall ed., New York, 2000.

H. Azizan, M. Jafarinasab, S. Behbahani, and M. Danesh. Fuzzy control based on LMI approach and fuzzy interpretation of the rider input for two wheeled balancing human transporter. 8th IEEE international conference on control and automation, pages 192-197, 2010. doi:10.1109/ICCA.2010.5524327.

C-H Huang, W-J Wang, and C-H Chiu. Design and Implementation of Fuzzy Control on a Two-Wheel Inverted Pendulum. IEEE Transactions on Industrial Electronics, volume 58(7):2988 - 3001, 2011. 